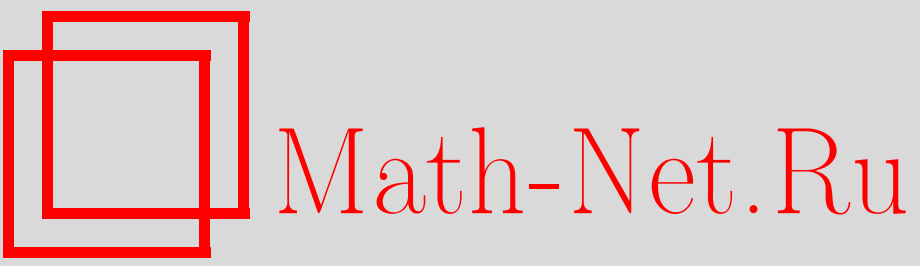

П. И. Пронин, К. В. Степаньянц, Инстантонный вклад в аномалию Rсимметрии, ТМФ, 1998, том 115, номер 3, 402-409

DOI: https://doi.org/10.4213/tmf882

Использование Общероссийского математического портала Math-Net.Ru подразумевает, что вы прочитали и согласны с пользовательским соглашением

http://www . mathnet.ru/rus/agreement

Параметры загрузки:

IP : 44.207 .124 .84

26 апреля 2023 г., 08:16:06 


\author{
ТЕОРЕТИЧЕСКАЯ \\ И МАТЕМАТИЧЕСКАЯ \\ ФИЗИКА \\ Том 115, № 3 \\ июнь, 1998
}

(C) $\mathbf{1 9 9 8}$ г.

П. И. Пронин* ${ }^{*}$ К.В. Степаньянц ${ }^{\dagger}$

\title{
ИНСТАНТОННЫЙ ВКЛАД \\ В АНОМАЛИЮ R-СИММЕТРИИ
}

Исследуется инстантонный вклад в аномалию R-симметрии $N=2$ суперсимметричной теории Янга-Миллса, связанный с неинвариантностью меры интегрирования по коллективным координатам. Результат явного вычисления одноинстантонной поправки согласуется с точным результатом. Производится качественное исследование многоинстантонных вкладов.

\section{1. ВВЕДЕНИЕ}

Роль непертурбативных вкладов в квантово-полевые аномалии, методы их вычисления и сравнения являются малоисследованной областью. До недавнего времени небольшое число статей (см. [1]) скорее просто указывало на принципиальную возможность существования таких вкладов. Так, в [1] было показано, что в теории Янга-Миллса инстантоны могут давать вклад в аномалии. Точный непертурбативный результат для $U(1)_{R}$-аномалии в $N=2$ суперсимметричной теории Янга-Миллса удалось получить косвенными методами [2] на основе результатов Зайберга и Виттена [3, 4]. В то же время до настояшего момента не было разработано и предложено регулярных способов вычисления непертурбативных вкладов, аналогичных методу Фуджикавы [5] в теории возмущений. Однако, по нашему мнению, непертурбативные методы могут существенно отличаться от него, т.к. физические эффекты, лежашие в их основе, принципиально иные.

Поскольку непертурбативный вклад в эффективное действие дают инстантоны [6-8], мы предлагаем в данной работе метод вычисления одно- и многоинстантонных вкладов, основанный на неинвариантности меры функционального интеграла.

Основное внимание уделено вычислению одноинстантонного вклада в аномалию R-симметрии в $N=2$ суперсиметричной теории Янга-Миллса. Показано, что эта аномалия связана с неинвариантностью меры интегрирования по коллективным координатам (как фермионным, так и бозонным). Для учета многоинстантонных вкладов предложен качественный анализ и показано, что вклад можно вычислить методами, аналогичными одноинстантонному примеру.

* Московский государственный университет, Москва, Россия. E-mail:pronin@theor.phys.msu.su

${ }^{\dagger}$ Московский государственный университет, Москва, Россия. E-mail:stepan@theor.phys.msu.su 
Статья организована следующим образом. В разделе 2 мы кратко напоминаем основные сведения о точных результатах в $N=2$ суперсимметричных теориях. В разделе 3 дается краткое изложение техники выгислений инстантонных вкладов в эффективное действие, обобшение и развитие которой, данное в разделе 4 , позволяет находить низшие непертурбативные поправки в аномалию. Здесь же вычислен одноинстантонный вклад в аномалию R-симметрии и показана его согласованность с точным результатом. Многоинстантонные поправки анализируются в разделе 5. В заключении кратко проанализированы основные результаты.

\section{2. $N=2$ СУПЕРСИММЕТРИЧНАЯ ТЕОРИЯ ЯНГА-МИЛЛСА}

$N=2$ суперсимметричная теория Янга-Миллса в суперпространстве описывается действием

$$
S=\frac{1}{16 \pi} \operatorname{tr} \operatorname{Im}\left(\tau \int d^{4} x d^{2} \theta_{1} d^{2} \theta_{2} \frac{1}{2} \Phi^{2}\right),
$$

где использованы следующие обозначения:

$$
\begin{gathered}
\Phi=-i e \Phi^{a} T^{a}, \quad \operatorname{tr} T^{a} T^{b}=\frac{1}{2} \delta^{a b}, \\
\tau=\frac{4 \pi i}{e^{2}}, \quad y^{\mu}=x^{\mu}+\frac{i}{2} \bar{\theta}_{i} \gamma^{\mu} \gamma_{5} \theta_{i}, \\
\Phi\left(y, \theta_{1}, \theta_{2}\right)=\phi\left(y, \theta_{1}\right)-i \bar{\theta}_{2}\left(1+\gamma_{5}\right) W\left(y, \theta_{1}\right)+\frac{1}{2} \bar{\theta}_{2}\left(1+\gamma_{5}\right) \theta_{2} G\left(y, \theta_{1}\right), \\
\phi\left(y, \theta_{1}\right)=P(y)+i S(y)+\bar{\theta}_{1}\left(1+\gamma_{5}\right) \psi_{1}(y)+\frac{1}{2} \bar{\theta}_{1}\left(1+\gamma_{5}\right) \theta_{1} f(y), \\
W\left(y, \theta_{1}\right)=\frac{1}{2}\left(1+\gamma_{5}\right) \times \\
\times\left(i \psi_{2}(y)+i \theta_{1} D(y)+\frac{1}{2} \Sigma_{\mu \nu} \theta_{1} F_{\mu \nu}(y)+\frac{1}{2} \bar{\theta}_{1}\left(1+\gamma_{5}\right) \theta_{1} \gamma^{\mu} D_{\mu} \psi_{2}(y)\right), \\
G\left(y, \theta_{1}\right)=\frac{1}{2} \int d^{2} \bar{\theta}_{1} e^{2 V} \phi^{+} e^{-2 V}, \\
V\left(x, \theta_{1}\right)=-\frac{i}{2} \bar{\theta}_{1} \gamma^{\mu} \gamma_{5} \theta_{1} A_{\mu}(x)+i\left(\bar{\theta}_{1} \theta_{1}\right)\left(\bar{\theta}_{1} \gamma_{5} \psi_{2}(x)\right)+\frac{i}{4}\left(\bar{\theta}_{1} \theta_{1}\right)^{2} D .
\end{gathered}
$$

Наиболее общий вид действия в теории с $N=2$ суперсимметрией есть

$$
\Gamma=\frac{1}{16 \pi} \operatorname{Im} \int d^{4} x d^{2} \theta_{1} d^{2} \theta_{2} F(\Phi),
$$

где функция $F$ назьвается препотенциалом.

В данной работе мы будем рассматривать только случай группы $S U(2)$, которая спонтанно нарушается до $U(1)$.

Действие (1) инвариантно относительно суперсимметричных и суперконформных преобразований

$$
\begin{aligned}
& \delta \Phi=\bar{\epsilon}_{i}\left(\frac{\partial}{\partial \bar{\theta}_{i}}+i \gamma^{\mu} \theta^{i} \frac{\partial}{\partial x_{\mu}}\right) \Phi, \\
& \delta \Phi=\bar{\beta}_{i} \gamma_{\nu} x^{\nu}\left(\frac{\partial}{\partial \bar{\theta}_{i}}+i \gamma^{\mu} \theta^{i} \frac{\partial}{\partial x_{\mu}}\right) \Phi .
\end{aligned}
$$


Инвариантность относительно преобразований из группы $U(1)_{R}$

$$
\Phi(\theta) \rightarrow e^{2 i \alpha} \Phi\left(e^{-i \alpha \gamma_{5}} \theta\right),
$$

которая в терминах компонентных полей переписывается как

$$
\begin{aligned}
P & \rightarrow P \cos 2 \alpha-S \sin 2 \alpha \\
S & \rightarrow S \cos 2 \alpha+P \sin 2 \alpha \\
\psi_{i} & \rightarrow\left(\cos \alpha+i \gamma_{5} \sin \alpha\right) \psi_{i}, \\
A_{\mu} & \rightarrow A_{\mu}
\end{aligned}
$$

порождает сохраняющийся ток $j_{R}^{\mu}$ :

$$
j_{R}^{\mu}=\bar{\psi}_{i}^{a} \gamma^{\mu} \gamma_{5} \psi_{i}^{a}-4 D^{\mu} P^{a} S^{a}+4 D^{\mu} S^{a} P^{a}
$$

Для фермионных полей $U(1)_{R}$ преобразования сводятся к киральным. Поэтому в рамках теории возмушений для группы $S U(2)$ аномалия тока $j_{R}^{\mu}$ будет

$$
\left\langle\partial_{\mu} j_{R}^{\mu}\right\rangle=\frac{e^{2}}{4 \pi^{2}} F_{\mu \nu}^{a} \widetilde{F}^{a \mu \nu}=-\frac{e^{2}}{16 \pi^{2}} \operatorname{Im} \Phi_{a}^{2} .
$$

В работе [2] данная аномалия была вычислена вне рамок теории возмушений. Было показано, что

$$
\left\langle\partial_{\mu} j_{R}^{\mu}\right\rangle=-\frac{1}{8 \pi^{2}} \operatorname{Im} \int d^{2} \theta_{1} d^{2} \theta_{2} u,
$$

где функция $u=u(\Phi) \rightarrow u(v)$ определяется из уравнения

$$
v(u)=\frac{\sqrt{2}}{e \pi} \int_{-1}^{1} d x \frac{\sqrt{x-u}}{\sqrt{x^{2}-1}}
$$

(здесь мы положили инстантонный масштаб $\Lambda=M \exp \left(-2 \pi^{2} / e^{2}\right)=1$ ).

Отметим, что формула (3) справедлива при вычислении как по теории возмушений, так и вне ее рамок. Результат теории возмущений имеет место в пределе $v \rightarrow \infty[3]$. В этом случае

$$
u=\frac{e^{2} \Phi^{2}}{2}+\frac{\Lambda^{4}}{4 e^{2} \Phi^{2}}+O\left(\frac{1}{\Phi^{6}}\right) .
$$

Первое слагаемое здесь правильно воспроизводит пертурбативный результат для аномалии, в то время как второе соответствует одноинстантонному вкладу. Компонентная запись второго слагаемого была получена в [2].

Высшие поправки могут быть определены из уравнения [4]

$$
\left(\frac{d^{2}}{d u^{2}}+\frac{1}{4\left(u^{2}-1\right)}\right) v=0
$$




\section{3. ИНСТАНТОНЫ И ДИНАМИКА ТЕОРИИ ПОЛЯ}

Непертурбативные поправки к эффективному действию связаны в первую очередь с инстантонными эффектами [7]. Поэтому вполне естественно предположить, что и непертурбативный вклад в аномалию также определяется инстантонами.

Техника вычислений инстантонного вклада в эффективное действие является хорошо известной $[9,10]$. Напомним кратко ее основные идеи.

В низшем приближении

$$
\Delta \Gamma=\frac{1}{Z_{0}} \int D \phi \exp \left(-S\left[\phi_{0}+\phi\right]\right)
$$

где $\phi$ обозначает весь набор полей теории, а $\phi_{0}$ - классическое решение евклидовых уравнений поля. Однако данное выражение является чисто формальным. Дело в том, что минимум евклидова действия, соответствующий инстантонным решениям, является вырож денным, т.к. действие преобразований симметрии на одно классическое решение дает новое с той же величиной действия. Формально это приводит к возникновению нулевых мод у второй вариации классического действия, которые должны удаляться интегрированием по соответствующим коллективным координатам. Мера интегрирования в несуперсимметричном случае впервые была вычислена 'т Хоофтом [9] и содержит вклады трансляционных (параметр $а$ ), масштабных $(\rho)$ и калибровочных $(\omega)$ нулевых мод (всего 8 коллективных координат). В $N$-суперсимметричном случае [11] добавляются еще $4 N$ нулевых мод ( $2 N$ из-за преобразований суперсимметрии и $2 N$ из-за суперконформных преобразований).

Явный вид нулевых мод может быть легко найден действием соответствующих преобразований на инстантонное решение. В частности, для фермионных нулевых мод при действии преобразований суперсимметрии получаем $(\epsilon \neq \epsilon(x))$

$$
\psi_{i 0}^{\text {susy }}=-2 \Sigma_{\mu \nu} \epsilon_{i} \frac{\rho^{2}}{\left(z^{2}+\rho^{2}\right)^{2}} \sigma_{\mu \nu},
$$

где $\sigma^{\mu \nu}=\eta^{a \mu \nu} \sigma_{a} / 2 ; \eta^{a \mu \nu}-$ символ 'т Хоофта, а

$$
z^{\mu}=y^{\mu}-a^{\mu}=x^{\mu}+\frac{i}{2} \bar{\theta}_{i} \gamma^{\mu} \gamma_{5} \theta_{i}-a^{\mu}
$$

в то время как суперконформные моды получаются заменой $\epsilon \rightarrow \gamma_{\alpha} z^{\alpha} \beta ; \beta \neq \beta(x)$ :

$$
\psi_{i 0}^{\mathrm{SC}}=-2 \Sigma_{\mu \nu} \gamma_{\alpha} z^{\alpha} \beta_{i} \frac{\rho^{2}}{\left(z^{2}+\rho^{2}\right)^{2}} \sigma_{\mu \nu} .
$$

$U(1)_{R}$-преобразования не дают новых нулевых мод, т.к. их действие на инстантонное суперполе сводится просто к изменению его параметров, и не выводят из пространства решений. 
В рассматриваемом случае $N=2$ суперсимметричной теории Янга-Миллса окончательное выражение для меры принимает вид

$$
d \mu=\text { const } \int d^{4} a \frac{d \rho}{\rho^{5}} \frac{d^{3} \omega}{2 \pi^{2}} M^{8} \rho^{8} \frac{1}{M^{4} \rho^{4}} \int d^{2} \epsilon_{1} d^{2} \epsilon_{2} d^{2} \bar{\beta}_{1} d^{2} \bar{\beta}_{2} .
$$

Следуюшим шагом при получении эффективного действия являются вычисление показателя экспоненты в пределе постоянного поля $[12,10,8]$ и взятие соответствуюшего интеграла. Однако для нас нет необходимости повторять его. Достаточно знать, что он может быть вьполнен и результат хорошо известен $[7,8]$.

\section{4. ИНСТАНТОННЫЙ ВКЛАД В АНОМАЛИЮ И НЕИНВАРИАНТНОСТЬ МЕРЫ}

В данном случае нам требуется вклад не в эффективное действие, а в аномалию

$$
\left\langle\partial_{\mu} j_{R}^{\mu}\right\rangle=-\left.\frac{\partial \Gamma}{\partial \alpha}\right|_{\alpha=0} .
$$

Для ее вычисления требуется только заметить [10], что показатель экспоненты является функцией от $\Phi\left(x^{\mu}=a^{\mu}, \theta_{i}=\epsilon_{i}+\gamma_{\alpha} x^{\alpha} \beta_{i}\right)$ и в низшем порядке по производным принимает вид

$$
\exp \left(-\frac{4 \pi^{2}}{e^{2}} \rho^{2}\left(1+2 \bar{\beta}_{i}\left(1+\gamma_{5}\right) \epsilon_{i}\right) \Phi^{2}\left(a^{\mu}, \epsilon_{i}+\gamma_{\alpha} x^{\alpha} \beta_{i}\right)\right) .
$$

Данное выражение не является $U(1)_{R}$-инвариантным, т.к.

$$
\begin{aligned}
\delta \Phi & =\left(1+2 i \alpha \gamma_{5}\right) \Phi, \\
\delta \epsilon_{i} & =\left(1+i \alpha \gamma_{5}\right) \epsilon_{i}, \\
\delta \beta_{i} & =\left(1+i \alpha \gamma_{5}\right) \beta_{i}, \\
\delta \rho & =0, \\
\delta a^{\mu} & =0 .
\end{aligned}
$$

Однако по нулевым модам производится интегрирование. Поэтому можно поступить следуюшим образом: сначала совершить $U(1)_{R}$-преобразования, а затем сделать замену

$$
\begin{aligned}
\theta & \rightarrow\left(1-i \alpha \gamma_{5}\right) \theta, \\
\epsilon & \rightarrow\left(1-i \alpha \gamma_{5}\right) \epsilon, \\
\beta & \rightarrow\left(1+i \alpha \gamma_{5}\right) \beta, \\
\rho & \rightarrow(1-2 i \alpha) \rho, \\
a^{\mu} & \rightarrow(1-2 i \alpha) a^{\mu},
\end{aligned}
$$


после которой показатель экспоненты восстанавливает свой прежний вид, но мера интегрирования при этом меняется:

$$
d \mu \rightarrow(1-2 i \alpha)^{4}(1-i \alpha)^{4}(1+i \alpha)^{4} d \mu=(1-8 i \alpha) d \mu .
$$

Необходимо только учесть, что при получении суперпотенциала остается интеграл

$$
\int d^{4} x d^{2} \theta_{1} d^{2} \theta_{2}
$$

(присутствуюший в определении суперпотенциала), в котором необходимо произвести обратную замену переменных. Однако при обратном преобразовании этот интеграл остается инвариантным, т.к. в результате двух проделанных вьше преобразований получаются замены

$$
x \rightarrow(1-2 i \alpha) x ; \quad \theta \rightarrow\left(1-2 \alpha \gamma_{5}\right) \theta .
$$

(Преобразования этого интеграла будут давать нетривиальный результат при рассмотрении $N=1$ суперсимметричных теорий.)

В итоге мы получаем, что

$$
\Gamma_{\text {inst }}^{\prime}=\int d \mu(1-8 i \alpha) e^{-S}=\frac{1}{16 \pi} \operatorname{Im} \int d^{4} x d^{2} \theta_{1} d^{2} \theta_{2}(1-8 i \alpha) \Delta F,
$$

где $\Delta F$ - инстантонный вклад в препотенциал, который вычислен в целом ряде работ (например, [8]) и в наших обозначениях имеет следуюший вид:

$$
\Delta F=\frac{1}{16 \pi} \frac{i \Lambda^{4}}{e^{2} \Phi^{2}}
$$

Остается только произвести дифференцирование по $\alpha$ и воспользоваться данными результатами. Окончательно имеем

$$
\left\langle\partial_{\mu} j_{R}^{\mu}\right\rangle_{1-\mathrm{inst}}=-\frac{1}{32 \pi^{2}} \operatorname{Im} \int d^{2} \theta_{1} d^{2} \theta_{2} \frac{\Lambda^{4}}{e^{2} \Phi^{2}},
$$

что полностью совпадает с первой нетривиальной поправкой к пертурбативному результату, полученной в [2] как асимптотика точного результата.

\section{5. МНОГОИНСТАНТОННЫЕ ВКЛАДЫ В АНОМАЛИЮ}

Исследуем теперь структуру высших инстантонных вкладов в аномалию R-симметрии. Для этого необходимо качественно исследовать структуру меры интегрирования по коллективным координатам.

Как мы вскоре увидим, наиболее важную роль играет количество фермионных коллективных координат, которое в суперсимметричных теориях есть не что иное, как количество фермионных нулевых мод. 
Количество нулевых мод оператора Дирака на топологически нетривиальном фоне определяется теоремой Атьи-Зингера

$$
n_{+}-n_{-}=\frac{1}{16 \pi^{2}} \operatorname{tr} \int d^{4} x t\left(F_{\mu \nu}\right) t\left(\widetilde{F}^{\mu \nu}\right)=\alpha_{t} n,
$$

где $\alpha_{t}$ - индекс Дынкина представления $t$, а $n$ - инстантонное число (для определенности далее $n \geqslant 0)$. Для группы $S U(2)$ сушествует единственный положительный корень, поэтому [13] имеем

$$
\alpha_{t}=\frac{1}{\operatorname{rank}(G)} \sum_{\text {weights }}(\lambda, \lambda)=\frac{1}{6}(2 j)(2 j+1)(2 j+2) .
$$

Здесь сумма берется по всем весам $\lambda$ представления $t$. В нашем случае фермионы находятся в присоединенном представлении, где $j=1$, и, следовательно, $\alpha_{t}=4$. Это соответствует двум суперсимметричным и двум суперконформным степеням свободы для каждого фермиона. В нашем случае имеются два фермиона. Поэтому полное число коллективных координат для $n$-инстантонного вклада есть просто $8 n$.

Каждая суперсимметричная нулевая мода дает вклад $1-i \alpha$ в меру интегрирования, а каждая суперконформная (без учета преобразования $\rho$ в мере) дает $1+i \alpha$, поэтому суммарный вклад равен единище.

Калибровочные нулевые моды (опять же без учета $\rho$ в мере), как мы видели, никакого вклада не дают. Поэтому необходимо лишь вычислить вклад размерных величин. Данная задача сушественно упрошается, если заметить, что все они имеют заведомо одинаковый закон преобразования типа $\rho \rightarrow(1-2 i \alpha) \rho$, в чем мы убеждаемся, рассматривая предел, когда все инстантоны находятся достаточно далеко друг от друга. Суммарная же размерность есть $4 n$, она получается при рассмотрении того же предела.

Все это приводит к следуюшему вкладу в аномалию:

$$
\frac{1}{16 \pi^{2}} \operatorname{Im}(-8 i n) \int d^{2} \theta_{1} d^{2} \theta_{2} \Delta F,
$$

где $\Delta F$ - инстантонный вклад в препотенциал.

Однако, с другой стороны, в силу (4) и (2) эта же величина есть

$$
\frac{1}{16 \pi^{2}} \operatorname{Im} \int d^{2} \theta_{1} d^{2} \theta_{2}\left(2 i \Phi \frac{\partial}{\partial \Phi}-4 i\right) \Delta F .
$$

Решая уравнение

$$
\Phi \frac{\partial}{\partial \Phi} \Delta F=(-4 n+2) \Delta F,
$$

находим, что инстантонное решение с топологическим числом $n$ дает поправку в препотенциал вида

$$
\Delta F=\operatorname{const} \Phi^{2(-2 n+1)},
$$


что находится в согласии с результатами [6], полученными на основе совсем других предположений. С помощью (5) легко найти, что вклад в аномалию имеет структуру

$$
\left\langle\partial_{\mu} j_{R}^{\mu}\right\rangle_{\text {inst }}=\operatorname{Im} \int d^{4} x d^{2} \theta_{1} d^{2} \theta_{2} \sum_{n=1}^{\infty} a_{n} \Phi^{2(-2 n+1)},
$$

где $a_{n}$ - вешественные коэффищиенты.

\section{6. ЗАКЛЮЧЕНИЕ}

В данной работе мы показали на примере $N=2$ суперсимметричной теории ЯнгаМиллса, каков вклад инстантонов в аномалии. Одноинстантонный вклад, полученный представленным методом, полностью согласуется с точным результатом, так же как и структура многоинстантонных поправок. Причина возникновения непертурбативных вкладов в аномалию, как оказалось, кроется в неинвариантности меры интегрирования по коллективным координатам, в отличие от неинвариантности меры относительно переменных интегрирования в методе Фуджикавы. Предложенный подход действительно сушественно отличается от последнего, поскольку принципиально отличаются физические причины возникновения аномальных вкладов.

Несомненный интерес представляет исследование непертурбативных вкладов в $N=1$ суперсимметричных полевых моделях, а также применение метода к другим типам аномалий и исследование аномалий реалистичных моделей. Мы вернемся к обсуждению этих вопросов в наших следуюших работах.

В заключение авторы выражают свою глубокую благодарность И. В. Тютину за ряд ценных замечаний и обсуждение результатов и А. А. Славнову за внимание к работе. Кроме того мы хотели бы особенно поблагодарить В.В. Асадова за финансовую поддержку исследований.

\section{Список литературы}

[1] V. Novikov, M. Shifman, A. Vainstein, V. Zakharov. Nucl. Phys. B. 1983. V. 229. P. 394.

[2] P. Howe, P. West. Nucl. Phys. B. 1997. V. 486. P. 245.

[3] N. Seiberg, E. Witten. Nucl. Phys. B. 1994. V. 426. P. 19.

[4] A. Bilal. Duality in $N=2$ SUSY $S U(2)$ Yang-Mills theory: A pedagogical introduction to the work of Seiberg and Witten. hep-th/9601007.

[5] K. Fujikawa. Phys. Rev. Lett. 1979. V. 42. P. 1979; 1980. V. 44. P. 1733.

[6] N. Seiberg. Phys. Lett. B. 1988. V. 206. P. 75.

[7] D. Finnell, P. Pouliot. Nucl. Phys. B. 1995. V. 453. P. 225.

[8] A. Yung. Nucl. Phys. B. 1997. V. 485. P. 38.

[9] G. 't Hooft. Phys. Rev. Lett. 1976. V. 37. P. 8; Phys. Rev. D. 1976. V. 14. P. 3432.

[10] M. Shifman, A. Vainstein, V. Zakharov. Nucl. Phys. B. 1980. V. 165. P. 45; V. Novikov, M. Shifman, A. Vainstein, V. Zakharov. Nucl. Phys. B. 1983. V. 229. P. 407; 1985. V. 260. P. 157.

[11] S. Cordes. Nucl. Phys. B. 1986. V. 273. P. 629.

[12] C. Callan, R. Dashen, D. Gross. Phys. Rev. D. 1978. V. 17. P. 2717.

[13] R. Slansky. Phys. Rep. 1981. V. 79. P. 1. 